\section{P348 PROVIDING COST-EFFECTIVE AND COORDINATED CARE FOR CHILDREN WITH MEDICAL COMPLEXITY}

Cristelle Chow*, Raveen Shahdadpuri. KK Women's and Children's Hospital, Singapore Singapore

\subsection{6/archdischild-2019-epa.695}

Introduction Many paediatric hospitals are treating increasing numbers of children with medical complexity (CMC), diagnosed with chronic life-limiting illnesses and requiring life-sustaining home medical technology. These medically fragile children and families are at risk of fragmented care, sub-optimal continuity and high healthcare resource utilization due to their multiple medical issues and care needs. Consequently, the Children's Complex and Home Care Services (CCHS) was established in our institution in April 2016 with the primary aims of providing coordinated, cost-effective and patient- and family-centered care to CMC and their families. This service is run by a multi-disciplinary team of pediatricians, nurses, allied health and administrative staff.

$\mathrm{CMC}$ in our context have a chronic life-limiting condition that involves at least three body systems and are often technologically dependent with limited mobility. In view of the numerous healthcare professionals involved in their care, multiple medical appointments are often scheduled which result in significant caregiver stress and fatigue. One of the key service implementations was multidisciplinary clinics whereby children are seen over the course of 1-3 hours by multiple clinical, nursing and allied health specialists. The purpose of this study is to describe CCHS service implementations, characterize CCHS patient characteristics and evaluate how multidisciplinary clinics have reduced their healthcare resource utilization.

Methods 55 patients who were enrolled in the CCHS between April 2016 and October 2018 were studied.

Results Patient ages ranged from 2 months to 14.3 years old at time of enrolment. The majority of patients had underlying primary genetic diagnoses (47.2\%), and other patients had either the primary diagnosis of cerebral palsy (20\%), congenital cardiac disease (5.4\%), neuromuscular disease $(3.6 \%)$ or another or undiagnosed underlying condition (23.6\%). Medical technology required at time of enrolment included enteral devices such as nasogastric/nasojejunal tubes or gastrostomies (94.5\%), suctioning machines (54.5\%), ventilator support $(34.5 \%)$ and tracheostomies (16.4\%).

CCHS multidisciplinary clinics managed to reduce the number of outpatient attendances by 6.8 visits per patient-year for $\mathrm{CMC}$ enrolled into the service. This saves caregivers from an equivalent number of workdays of lost salary, and translates to $€ 450$ of savings per patient per year on just transportation costs alone.

Conclusion CMC are heterogeneous in conditions but similar in care needs, and reducing outpatient attendances and healthcare costs is possible with coordinated multi-disciplinary clinics.

\section{P349 'UNRAVELLING THE DNA' - AUDIT OF NON- ATTENDANCE AT GENERAL PAEDIATRIC OUTPATIENT CLINICS}

Aoife Cassidy*, Joanne Beamish, Juliette Lucey, Eimear Kelly. Temple Street Children's University Hospital, Dublin, Ireland

10.1136/archdischild-2019-epa.696
Aim 'Did not attends' (DNAs) are a frequent occurrence at outpatient clinics. These affect waiting list times, cost the hospital money, and may mean children are not receiving the medical care that they require. The Health Service Executive (HSE) DNA targets for 2016 were 12\% for new attendance DNA rates ${ }^{1}$, and for $70 \%$ of consultants to have a DNA rate of $<10 \%$. This study aimed to audit current DNA rates in two General Paediatric clinics, and also to identify reasons for non-attendance and possible ways to overcome these.

Method The scheduled patients and non-attenders of two General Paediatric clinics were included from January to April 2017. Parents of children who did not attend were contacted to participate in a telephone questionnaire. These responses were analysed.

Results Twenty-two clinics were included, amounting to 329 patients. There were 59 (17.93\%) DNAs giving an average of 2.68 DNAs per clinic. There was no significant difference in DNA rates between sexes, appointment times or new vs return patients. There were higher DNA rates in children aged 11 or older.

Twenty-seven $(45.76 \%)$ parents responded to the questionnaire. Thirty $(51 \%)$ parents' contact numbers were not working or unavailable. The most common reasons for not attending were: not aware had appointment $(n=9,33 \%)$, child now well $(n=4,15 \%)$, parent working $(n=3,11 \%)$. Many parents suggested appointment times outside of office-hours or on Saturdays so their child would not miss school. Parents prefer text as method of appointment reminder. Each DNA cost the hospital $€ 250$.

Conclusion 'Did not attends' are a frequent occurrence at General Paediatrics clinics. This is costly and affects waitinglist times. Children are dependent on their caregiver to bring them to outpatient appointments. Potential solutions are multiple reminders, appointment times outside of office hours and a hospital appointment mobile phone app. Lack of up-to-date caregiver contact details is a major issue to be overcome.

\section{P350 CHILDREN DISCHARGED DIRECTLY HOME FROM THE PAEDIATRIC INTENSIVE CARE UNIT}

Roy Gavin Stone*, Laura Mac Darby, Bryony Treston, Suzanne Crowe. Paediatric Intensive Care Department, Our Lady's Hospital For Sick Children Crumlin, Dublin, Ireland

\subsection{6/archdischild-2019-epa.697}

Background The process of discharging a patient directly home from the paediatric intensive care unit is distinctly different from discharging a patient to a hospital ward or another hospital. Whether these differences increase the risk of communication errors or increased mortality has not been previously investigated in a paediatric cohort. For adult populations the discharge of select patients directly home from the intensive care unit is not associated with increased healthcare utilisation or increased mortality. Whether discharge directly home from the Paediatric Intensive Care Unit is associated with increased mortality has not been previously investigated in the literature.

Aim To describe cases presenting for admission to the Paediatric Intensive Care Unit (PICU) but who are sufficiently recovered to be discharged directly home.

Methods This single centre retrospective descriptive study was conducted from the 1st July 2012 to 1st July 2018. Data was extracted from the PICU Clinical Information Portfolio system. 
Patients with a palliative diagnosis were excluded from this study.

Results 87 patients were discharged directly home in the 6 year study period. No patients died within 30 days of discharge. The median PIM 3 score was 0.0139 (IQR 0.00720.0347). Of patients admitted diagnoses were $41 \%$ Respiratory, 33\% Peri-operative, 8\% Cardiology, 6\% Toxicology, 5\% Neurology, 5\% Gastrointestinal, 2\% Allergy and 1\% Trauma. $69(79 \%)$ of patients had a past medical history. In 34 (39\%) admissions patients had a tracheostomy. There were no discharges against medical advice. The primary team was informed of discharge for 84 (97\%) patients. A documented follow-up plan was recorded for 46 (53\%) patients. There was written communication with the patients general practitioner for $22(25 \%)$ patients. $5(6 \%)$ patients were discharged outside the hours of 9:00-17:00.

Conclusions For the most part primary teams were informed and the majority of patients had follow-up arranged, however communication with the GP was poor. Following the results of this study a standardised template was introduced at our institution to improve the discharge process.

\section{P351 THE ESTABLISHMENT OF A PAEDIATRIC METABOLIC OUTREACH CLINIC AT UNIVERSITY HOSPITAL LIMERICK}

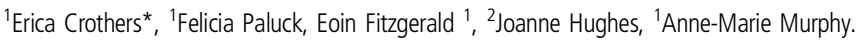
${ }^{1}$ Department of Paediatrics, University Hospital Limerick, Limerick, Ireland; ${ }^{2}$ National Centre for Inherited Metabolic Disorders, Temple Street Children's Hospital, Dublin, Ireland

\subsection{6/archdischild-2019-epa.698}

Background and aims Paediatric Medicine in Ireland is growing and changing. Care is now being delivered in alliance with the 'Hub and Spoke' model of care. Since the introduction of Newborn Screening in Ireland in 1966 there has been an increasing need for metabolic specialist care. The National Centre for Inherited Metabolic Disorders (NCIMD) is located at the Children's University Hospital, Temple Street and provides care for patients with Inherited Metabolic Disorders (IMDs). Due to the complexity of IMDs, multidisciplinary care is essential and is not only provided via Temple street, but now also through Pediatric Outreach Clinics.

The aim of this study was to describe the establishment of the Pediatric Metabolic Outreach Clinic (PMOC) at University Hospital Limerick (UHL).

Methods We describe the process involved in the establishment of the clinic, its' location and structure and the type and number of patients seen.

Results Paediatric patients with a formal diagnosis of an IMD or who were under investigation for such a diagnosis residing within the UHL catchment area were offered a review in the MOC in the Paediatric Outpatient Department at UHL in place of one of the reviews at the tertiary centre. The appointments are offered by and at the discretion of the NCIMD on a rotational basis to allow all patients living in the region the opportunity to attend and thus reduce the frequency of travel to Dublin.

The PMOC comprised a full day clinic including consultation with the Metabolic Paediatrician, Specialist Dietician and Nurse.

Since 2013 there have been 15 PMOCs at UHL.

Patients with a variety of IMDs were seen.

Conclusion With the increasing demand on Specialist Services in Ireland, outreach clinics are paramount for the future care of Paediatric patients ${ }^{4}$. Most importantly, those with IMDs in the Midwest are able to access the highest level of care closer to home.

\section{P352 A PRISM OF SELF-REFLECTION; EXPLORING THE EFFECTS OF PHYSICAL EXERCISE, REST, SOCIAL TIME, AND MINDFULNESS ON STAFF HEALTH AND WELLBEING}

${ }^{1}$ Sean Casey*, 'Jonathan Clarke, ${ }^{1}$ Alan Finan, ${ }^{2}$ Paul Gaffney, ${ }^{2}$ Aisling Hagerty. ${ }^{1}$ Paediatric Department, Cavan General Hospital, Cavan, Ireland; ${ }^{2}$ Cavan/Monaghan Primary Care Psychology Service, Cavan, Ireland

\subsection{6/archdischild-2019-epa.699}

Background There is an established body of evidence that medical professionals are at increased risk of poor mental and physical health. This negatively affects the individual practitioner, the healthcare system, and patient outcomes. To date, there have been limited interventional studies in Ireland to improve hospital staff wellbeing.

Study Design Prospective interventional study

Methods Participation was open to all staff in the paediatric department of Cavan General Hospital, a level 2 hospital in rural Ireland. A 10-week Wellness Diary was designed, in which participants recorded their weekly physical exercise, sleep hygiene, social time, and mindfulness practice.

Targets for each parameter were provided. The authors organised events and issued weekly motivational emails to help participants meet their targets. The Perceived Stress Scale (PSS) and Pittsburgh Sleep Quality Index (PSQI) were used to measure stress levels and sleep quality at baseline, Week 5, and Week 10.

Data was analyzed using Microsoft Excel 2011. Spearman coefficient was used to assess for statistically significant correlations between variables.

Results 43 participants were initially enrolled. 29 submitted diaries at Week 5, and 20 submitted at Week 10.

$55 \%$ of all participants were compliant with physical exercise targets at baseline, which remained unchanged at Week 10.

$49 \%$ of participants $(n=21)$ reported PSQI scores of $\leq 5$ (indicative of good quality sleep) at baseline, versus 59\% ( $\mathrm{n}=$ 17) by week 5 , and $89 \%(n=17)$ by week 10 . Of participants who completed the programme, $63 \%$ had PSQI scores $\leq 5$ at baseline, $68 \%$ by week 5 , and $89 \%$ by week 10 . This correlated weakly with compliance with sleep hygiene targets, though this was not statistically significant.

At baseline, participants reported having enough time for family/personal life 4.88 days of the week. This had increased to 5.05 days of the week by Week 10 .

$35 \%$ of participants $(n=15)$ used weekly mindfulness practice at baseline, versus $66 \%$ at Week 5 , and $70 \%$ at Week 10 . Of the participants who completed the programme, mean PSS scores at baseline were 15.7, 15.8 at Week 5, and 13.7 at Week 10. This correlated weakly with increased mindfulness use, though the correlation was not statistically significant.

Conclusions There are a number of limitations to our study. Only 20 participants completed all 10 weeks of the programme, limiting result generalisation. To the authors' knowledge, this is the first study of its kind in Ireland and its findings may influence future interventions. 